\title{
Epidemiology of Binge Eating Disorder
}

\author{
Ruth H. Striegel-Moore ${ }^{1 *}$ and Debra L. Franko ${ }^{2}$ \\ ${ }^{1}$ Department of Psychology, Wesleyan University, Middletown, Connecticut \\ ${ }^{2}$ Department of Psychology, Northeastern University, Boston, Massachusetts
}

Accepted 28 March 2003

\begin{abstract}
Objective: First described over 50 years ago, binge eating disorder (BED) only recently has become the focus of epidemiologic studies. This article provides a comprehensive review of these studies. Method: Relevant studies were examined and summarized in the form of a narrative review. Results: Similar to the early studies of bulimia nervosa (BN), the first generation of epidemiologic studies of BED is limited in scope or methodology. They focus on prevalence rates and provide only basic demographic characteristics and often use less than optimal sampling or assessment methods. Discussion: Results suggest that the demographic profile of BED may be more diverse than that of BN. Future studies should evaluate complex etiologic models in representative samples that include men and a broader range of ethnic minority groups. (c) 2003 by Wiley Perodicals, Inc. Int J Eat Disord 34: S19-S29, 2003.
\end{abstract}

Key words: epidemiology; diverse demographic profile; obese patients; binge eating

\section{INTRODUCTION}

Early clinical descriptions suggested that a distinct subgroup of obese patients evidenced recurrent binge eating but did not purge (Stunkard, 1959). The phenomenon of binge eating in the absence of the inappropriate compensatory behaviors that define bulimia nervosa $(\mathrm{BN})$ attracted relatively little scientific interest until the introduction of binge eating disorder (BED). BED was introduced in the 4th ed. of the Diagnostic and Statistical Manual of Mental Disorders (DSM-IV; American Psychiatric Association, 1994) as a specific example of eating disorder not otherwise specified (EDNOS) and as a provisional diagnosis that is distinct from BN. In the past decade, a rapidly growing literature has documented the clinical significance of BED in terms of the health and mental health problems commonly reported by individuals who meet criteria for this syndrome (Wilfley, Wilson, \& Agras, 2003). With remarkable consistency, studies of

*Correspondence to: Dr. Ruth H. Striegel-Moore, Department of Psychology, Wesleyan University, 207 High Street, Middletown, CT 06459. E-mail: rstriegel@wesleyan.edu

Grant sponsor: National Institute of Mental Health and the National Institute of Diabetes, Digestive and Kidney Disease; Grant number: MH 57897.

Published online in Wiley InterScience (www.interscience.wiley.com). DOI: 10.1002/eat.10202

(C) 2003 by Wiley Periodicals, Inc. 
patient samples have found very high rates of obesity and psychiatric comorbidity (especially mood and anxiety disorders; for reviews, see de Zwaan, 2001; Walsh \& Devlin, 1998). Several important treatment studies have been published that provide answers to the question of how best to treat this disorder (for reviews, see Carter, Hudson, Lalonde, Pindyck, \& Pope, 2003; Wonderlich, de Zwaan, Mitchell, Peterson, \& Crow, 2003).

Epidemiologic research often lags behind research that describes the clinical picture or tests and therapeutic interventions (Striegel-Moore \& Cachelin, 2001). As the current review will show, research of the epidemiology of BED is in the early stages.

Epidemiologic studies focus on two related questions: (1) How many individuals in a population meet diagnostic criteria for the disorder of interest? (2) Who in the population in particular is likely to meet criteria for the disorder? The epidemiology of BED has a relatively short history. Therefore, answers to either question are quite preliminary. First, we review studies that provide estimates of the prevalence of BED. Many of these studies also describe correlates of BED, most commonly demographic characteristics, as well as the rates of obesity. We then focus more specifically on the demographic correlates of BED. Fixed markers (i.e., risk factors that cannot be changed; Kraemer et al., 1997), such as gender and ethnicity, are of interest because they identify high-risk groups in the population. A few reports described variable risk factors (Fairburn et al., 1998; Striegel-Moore, Dohm, Pike, Wilfley, \& Fairburn, 2002), but the studies were preliminary and, therefore, were omitted from consideration in this review. We conclude with comments regarding the future directions in this area of research.

\section{STUDIES OF THE PREVALENCE OF BED}

Given the relatively recent introduction of BED into the psychiatric nomenclature, epidemiologic research is limited. Several epidemiologic studies in North America described nonpurging bulimia, which is characterized by alternating periods of binge eating and dieting without purging behaviors (Bruce \& Agras, 1992; Garfinkel et al., 1995; Kendler et al., 1991; Lewinsohn, Striegel-Moore, \& Seeley, 2000). These studies included individuals who, by the more specific current definitions of $\mathrm{BN}$ and BED, might meet BED criteria. In addition, some of the individuals in these studies were classified as "spectrum $\mathrm{BN}^{\prime}$ " because they did not meet the criterion of inappropriate compensatory behaviors. However, using DSM-IV criteria, they might best be described as BED cases. Although these early epidemiologic studies did not assess participants specifically for BED, they are important because they set the methodologic standard. They used representative samples and two-stage assessments by research interviews (rather than selfreport questionnaires) with clearly defined diagnostic algorithms. Several reviews have concluded that the prevalence of $\mathrm{BN}$ in adult women ranges from $1 \%$ to $3 \%$. Studies of younger age groups or more recent cohorts report higher estimates than studies that are based on samples that span a broad age spectrum (Fairburn \& Beglin, 1990; Hoek, 2002). Inclusion of male participants is uncommon in epidemiologic studies of BN. Experts estimate that female cases outnumber male cases by a factor of 10 (Garfinkel et al., 1995). Although this review focuses on BED, we will note the prevalence of BN when data are available.

Epidemiologic studies of BN consistently have shown that assessment strategies influence prevalence results (Hoek, 2002). One important source of measurement error is the ambiguous meaning of "binge eating" when used by the lay public. The DSM-IV 
definitions of $\mathrm{BN}$ and $\mathrm{BED}$ require that eating episodes are considered binge eating only if they involve eating an unusually large amount of food ("objective overeating") and a sense of loss of control over the eating episode ("loss of control"). Research has shown that study respondents do not necessarily invoke either the objective overeating or the loss of control criterion when answering affirmatively to the question of whether they have experienced episodes of binge eating (Beglin \& Fairburn, 1992; Williamson, Gleaves, \& Lawson, 1991). In self-report measurements, more individuals meet criteria for binge eating than in interview-based assessments (Fairburn \& Beglin, 1990, 1994) because the latter permit clarification of the meaning of binge eating and rescoring of affirmative answers to negative answers if the episode was not objectively large or experienced as out of control.

Despite the methodologic limitations of self-report questionnaires, we include studies that report information on the prevalence of BED. In the early stage of BED research, these studies served the important function of generating new hypotheses.

\section{Prevalence Studies Using Self-Report Instruments}

To support the newly defined disorder, Spitzer et al. $(1992,1993)$ conducted BED field trials among several community and patient samples. Although recruited at various sites in the United States, these samples represented "convenience" rather than representative samples. In addition, they relied on self-report questionnaires to identify BED cases, a method that yields a considerable number of false-positive cases (Fairburn \& Beglin, 1990). In the initial field trial (Spitzer et al., 1992) of a community sample of 1,031 males and females (62\% female, 36\% minorities, mean age 33 years), $2.5 \%$ of female respondents and $1.1 \%$ of male respondents met criteria for current BED (this gender difference was not statistically significant). In addition, $1.5 \%$ of respondents (gender not specified) met criteria for BN. Among 723 patients recruited at weight loss or eating disorder programs ( $81 \%$ female, $11 \%$ minorities, mean age 44 years), significantly more women $(31.9 \%)$ than men $(20.8 \%)$ met criteria for BED. No breakdown was provided by ethnicity for either sample. Finally, the authors noted that in the weight control sample, a significantly greater proportion of individuals with BED (62.8\%) versus those without BED (44.9\%) were at or exceeded a body mass index (BMI) of 35. In the community sample, results were evaluated using a different cut-off for obesity (BMI $=27.5$ or greater). Spitzer et al. (1992) noted that only one half of the community cases with BED $(n=8)$ met criteria for this obesity threshold and no comparison data were provided for the non-BED community participants.

The second field trial (Spitzer et al., 1993) reported that among 728 college students (79\% female, $26 \%$ minorities, mean age 22 years), $2.8 \%$ of women and $1.9 \%$ of men met criteria for BED. In addition, $1.2 \%$ of the female students met criteria for BN (it is unclear if there were any male BN cases). In the community sample of 214 employees at a large medical center (70\% female, $41 \%$ minorities, mean age 34 years), 5.3\% of women and $3.1 \%$ of men met BED criteria and $0.5 \%$ of BN cases were detected (gender not specified). The gender differences in the prevalence of BED in the college or community sample did not reach statistical significance. Data were not reported separately for White versus minority groups. Among 1,785 participants of weight control programs recruited at 18 different programs (89\% female, $8 \%$ minorities, mean age 43 years), $29.7 \%$ of female patients and $21.8 \%$ of male patients $(p<.02)$ met BED criteria. Non-White patients enrolled in weight loss programs, most of whom were Black, were as likely to meet criteria for BED (22\%) as were White patients (29\%). Spitzer et al. (1993) again noted 
a significant association between BED and obesity (BMI $=35$ or greater), but limited their data report to the weight control sample. Finally, they acknowledged that a differential diagnosis of BED versus nonpurging BN might be difficult using the field study's selfreport questionnaire. The authors indicated that a refined estimate, excluding possible nonpurging BN cases from the case count, decreased the BED prevalence by $12 \%$.

Despite the significant methodologic limitations inherent in recruiting samples of convenience and relying solely on self-report questionnaires to establish the eating disorder diagnosis, these early studies gained considerable attention. They provided the first effort at describing BED and they were among the first large-scale surveys to examine eating disorders in male and in non-White populations. Typically, eating disorders were characterized as being limited to White females (for review, see StriegelMoore \& Smolak, 2000) and the BED field trials results suggested that, at least for BED, this prevailing view might need to be reconsidered.

Using the self-report instrument that had been developed for the BED field trials, Smith, Marcus, Lewis, Fitzgibbon, and Schreiner (1998) evaluated BED in an epidemiologic study of 1,837 men (45\% Black) and 1,895 women (51\% Black; sample age range 28-40 years) and found an overall prevalence rate of $1.5 \%$. There were, however, significant differences in the prevalence rates across the race-gender groups. BED rates did not differ significantly among Black women (2.2\%) compared with White women $(2.0 \%)$ and rates observed among White men (1.2\%) did not differ significantly from those obtained for White women. In contrast, Black men $(0.4 \%)$ had significantly lower BED rates than the other groups. Rates of BED among overweight (BMI = 27.5 or greater) individuals $(2.9 \%)$ were almost double the rates observed in the overall cohort. This study further reinforced the view that BED may affect a demographically more diverse group than $\mathrm{BN}$ and may be more frequently found in obese than nonobese groups.

In a study of 507 male and female social work students (mean age 26 years) in Germany (Thiels \& Garthe, 2000), 3.8\% of women and 3.5\% of men fulfilled DSM-IV criteria for BED, based on the Bulimic Investigatory Test Edinburgh (BITE; Henderson \& Freeman, 1987). However, the BITE was not designed initially to measure BED and is not a widely used instrument. The relatively high prevalence rates observed in this sample may reflect the limited number of specific questions directed at the diagnostic criteria for BED.

Some studies have evaluated rates of eating disorders among patients recruited in primary care (Linzer et al., 1996; Spitzer, Kroenke, \& Williams, 1999), obstetric-gynecology practice (Spitzer, Williams, Kroenke, Hornyak, \& McMurray, 2000), or general hospital settings (Diez-Quevedo, Rangil, Sanchez-Planell, Kroenke, \& Spitzer, 2001). Diagnoses were based on the PRIME-MD Patient Health Questionnaire (PHQ; Spitzer et al., 1994), a self-report screening instrument for mental disorders that includes diagnostic items for $\mathrm{BN}$ and BED. In a sample of 559 women (48\% minorities) and 382 men (34\% minorities) recruited in primary care practices (mean age 55 years), $4 \%$ of women and $2 \%$ of men met criteria for current eating disorder involving binge eating (i.e., either BN or BED, no further details provided; Linzer et al., 1996). Despite the large number of minority patients included in this study, data were not reported by ethnicity. A subsequent study (Spitzer et al., 1999) of a sample of 3,000 patients (66\% female; $21 \%$ minorities, mean age 46 years) recruited at family medicine or internal medicine practices found that $6 \%$ met criteria for current BED (and 1\% for current BN). No further demographic information was reported about the BED cases. In a sample of 3,000 women (mean age 31 years) recruited at seven obstetric-gynecologic outpatient centers (where ethnic representation rates varied from a low of less than 3\% to a high of greater than $90 \%$ ), $4 \%$ of participants met criteria for current $\mathrm{BED}$ (and $1 \%$ met criteria for $\mathrm{BN}$ ). BED rates were not reported by ethnicity 
(Spitzer et al., 2000). Finally, in a study of 1,003 female and male general hospital patients recruited in Spain (54\% male, sample mean age 43 years), the rate for current BED was $5.3 \%$ and $1.2 \%$ for BN (gender not specified; Diez-Quevedo et al., 2001).

The prevalence rates reported in these four studies (Diez-Quevedo et al., 2001; Linzer et al., 1996; Spitzer et al., 1999, 2000) are difficult to interpret relative to rates observed in the general population because of the well-established fact that psychiatric disorders in general are more common among primary care patient samples than in the general population (Kessler et al., 1999). The samples included in these studies (Diez-Quevedo et al., 2001; Linzer et al., 1996; Spitzer et al., 1999, 2000) are older than the samples in the other survey studies (Smith et al., 1998; Spitzer et al., 1992, 1993; Thiels \& Garthe, 2000) described so far in this review. Although in studies of BN, increasing age of the study sample is associated with decreasing rates of BN (Kendler et al., 1991), Johnson, Spitzer, and Williams (1999) have reported that rates for BED increase with increasing age of the study sample. The relatively high prevalence rates reported in these four studies may reflect both the inclusion of a relatively older age group and the biases inherent in studying patient samples.

These four studies (Diez-Quevedo et al., 2001; Linzer et al., 1996; Spitzer et al., 1999, 2000) are noteworthy for their inclusion of a large population of men and ethnic minority groups, both of which are underrepresented in eating disorder research. Based on the PHQ, rates for BED among male patients in primary care settings are significantly lower than those reported for female patients. An important contribution to the literature of these studies are the data they provided on the operating characteristics of the PHQ. A comparison of eating disorder diagnoses based on the PHQ to those based on clinical assessments by mental health professionals suggests a satisfactory predictive value (overall accuracy $94 \%-98 \%$, sensitivity $75 \%-92 \%$, specificity $90 \%-98 \%$, and a Cohen's kappa value of 0.65-0.79; Diez-Quevedo et al., 2001; Spitzer et al., 1999). The overall accuracy of any eating disorder diagnosis using the PHQ (relative to expert clinical interview) compared favorably with the overall accuracy of any mood disorder $(85 \%-91 \%)$ or any anxiety disorder $(89 \%-93 \%)$. If investigators are seeking to make a differential of BED versus other specific eating disorders, the accuracy estimates might be lower. Most notably, the distinction between BED and nonpurging BN might be more difficult to make compared with the distinction between any eating disorder and no eating disorder. These encouraging findings regarding the validity of the PHQ await replication in studies by other investigators. If replicated, these results would support the use of the PHQ for screening purposes.

Because so few studies have examined the prevalence of eating disorders involving binge eating in male samples, one additional study warrants review even though it does not report data on full-syndrome BED. The Oregon Adolescent Depression Project (OADP), which was initiated before BED had been defined, was one of a small set of epidemiologic studies to report prevalence rates for BN not only in females but also in males (Lewinsohn, Hops, Roberts, Seeley, \& Andrews, 1993). Consistent with results obtained in other epidemiologic studies, lifetime rates for BN were significantly higher in girls $(1.6 \%)$ than in boys $(0.14 \%)$. A subsample of the OADP participants has been followed and a self-report survey conducted after the participants had reached their 24th birthday included questions about eating disorders symptoms (Lewinsohn, Seeley, Moerk, \& Striegel-Moore, 2002). Among the young adult men and women (mean age 25 years), men (28\%) were significantly more likely than women $(14 \%)$ to report that they had experienced episodes of overeating. The questionnaire asked respondents to describe two typical overeating episodes. These were evaluated by two raters to determine 
whether the amounts described were objectively large as defined by the scoring criteria developed for the Eating Disorders Examination (EDE; Fairburn \& Cooper, 1993). Based on these ratings, $32 \%$ of male and $42 \%$ of female overeaters reported episodes that did not meet the objective overeating criterion (a nonsignificant gender difference). Among the overeaters whose episodes did meet the objective overeating criterion, women were significantly more likely than men to report loss of control over (68\% vs. $27 \%$ ) or distress about the eating episode ( $89 \%$ vs. $38 \%$ ). When taking into account both the amount of food and the loss of control criterion for the definition of binge eating, $2.6 \%$ of the men and $4 \%$ of women reported binge eating at least twice per week in the preceding 3 months $(p>.05)$. Hence, when starting out with a survey response to a question about overeating, more men than women were identified. Adding the requirements of an objectively large amount of food to be consumed and loss of control to be experienced over the eating episode greatly reduced the overall number of respondents who met criteria for binge eating. The gender difference in the rate of binge eating was not significant. Binge eating was associated with overweight in both men and women, which supports the view that binge eating is clinically significant. Male binge eaters were significantly less likely than female binge eaters to report inappropriate compensatory behaviors. The gender differences regarding compensatory behaviors and distress about binge eating suggest that men might be at lower risk for full-syndrome eating disorders compared with women because men experience their disordered eating differently.

\section{Prevalence Studies Using Interview Methods}

Although it did not evaluate rates of BED, the interview-based study of eating disorders conducted by the Toronto group before BED was defined provided an important early report about disordered eating in men (Garfinkel et al., 1995; Woodside, Garfinkel, Lin, Goering, \& Kaplan, 2001). In this representative population sample of 8,116 adolescent (older than 14 years) and adult Canadians, rates for full-syndrome BN were significantly lower among males $(0.13 \%)$ than females $(1.46 \%)$. However, recurrent binge eating (more than twice per week) was equally common in men $(3.2 \%)$ and women $(3.3 \%)$. Binge eating (determined by interview response and consistent with the DSMIV definition), the entry criterion for a diagnosis of either BN or BED, was fulfilled by comparable numbers of men and women. Male binge eaters were significantly less likely than female binge eaters to report using extreme compensatory behaviors to counteract the effects of binge eating, possibly accounting for the significantly lower rate of BN among men (Garfinkel et al., 1995). Unfortunately, it is unclear how many of the recurrent binge eaters also had the additional symptoms required for a diagnosis of BED.

More recent epidemiologic studies have been conducted with large samples. For example, a representative sample of 1,000 women (mean age 39.6 years; range 15-85 years) in Tyrol, Austria, was screened by telephone interview (Kinzl, Traweger, Trefalt, Mangweth, \& Biebl, 1999a). Kinzl et al. reported a BED point-prevalence rate of 3.3\%, and $1.5 \%$ of the women met criteria for current BN. Rates of BED were higher in overweight $(4.1 \%)$ or obese $(8.5 \%)$ women than in normal weight women $(2.9 \%)$. This difference was not statistically significant $(p=.075)$, although the sample may have been too small to detect significant effects. The authors also evaluated prevalence rates for BED and BN by age groups and found that BED was distributed evenly across the age groups younger than 25 years, 25-34 years, 35-44 years, 45-54 years, and 54-64 years. In contrast, Garfinkel et al. (1995) and Kendler et al. (1991) reported that the prevalence of BN was 
highest among women younger than 25 years old $(6.2 \%)$ and decreased to $1.5 \%$ (in the 25-34 age group) or less than 1\% among the older women. In another report, Kinzl, Traweger, Trefalt, Mangweth, and Biebl (1999b) described results of a similar study conducted among a representative sample of 1,000 Tyrolean men (18-88 age group). Current BED or current BN was identified in $0.8 \%$ and $0.5 \%$, respectively. Although the screening questions asked about eating unusually large amounts of food, it is unclear whether the interviewers actually ascertained enough detail about the eating episodes to determine whether the amounts were objectively large (Fairburn \& Cooper, 1993). This may account for the relatively high rates of BED in the female sample and the extraordinarily high rates of current $\mathrm{BN}(6.2 \%)$.

In a representative community sample of 1,785 women and 2,725 men recruited in South Australia (mean age 46 years), Hay (1998) reported a combined rate of 1\% for BED (and $0.26 \%$ for BN) using the EDE (Fairburn \& Cooper, 1993), a structured, investigatorbased interview. No information was provided regarding the gender of the individuals with an eating disorder diagnosis. Hay (1998) also found that regular binge eating (a minimum average of once per week) was as common among women (3.4\%) as among men $(3 \%)$ and was associated with elevated body weight in both genders.

Although the New England Women's Health Study (NEWHP; Striegel-Moore, Wilfley, Pike, Dohm, \& Fairburn, 2000) did not determine the prevalence of full-syndrome BED, this study is included here because it provides data on binge eating problems in Black women, an underresearched population group. In this community sample of 1,628 Black and 5,741 White women, binge eating at least twice per week during the preceding 3 months, as determined by an EDE-based, telephone-administered interview, was reported by more Black women (4.5\%) than White women $(2.6 \%)$. In both ethnic groups, recurrent binge eating was associated significantly with an elevated BMI and with elevated rates of self-reported psychiatric symptoms. A subsequent study based on women who met full-syndrome criteria for BED reported an association between BMI and BED in both Black and White women (Pike, Dohm, Striegel-Moore, Wilfley, \& Fairburn, 2001). Specifically, $83 \%$ of Black women and $56 \%$ of White women with BED were obese (BMI > 30), compared with $35 \%$ of Black women and $13 \%$ of White women without a diagnosis of BED. These studies suggest the need for inclusion of Black women in studies of BED.

\section{THE DEMOGRAPHICS OF BED}

Demographic characteristics of individuals with BED have focused on gender and ethnicity. To our knowledge, there are no published reports of additional variables such as socioeconomic status (SES) or geographic diversity.

\section{Gender and BED}

Several studies did not find significant gender differences when comparing White women and men on rates of BED. Among primary care patients in the United States, men were significantly less likely than women to meet criteria for BED (Linzer et al., 1996). However, Linzer et al. did not test specifically for the Gender $\times$ Ethnicity interaction. It is unclear whether the differences in results between White men and White women might no longer have been significant if the Gender $\times$ Ethnicity interaction had been considered (Smith et al., 1998). The relatively attenuated gender difference in BED 
raises the question: How do BED and $\mathrm{BN}$ differ in risk factors? Risk factor models for $\mathrm{BN}$ have emphasized gender as an important fixed marker (Striegel-Moore \& Cachelin, 2001) and have specified gender-specific biologic (e.g., differences in serotonin response to dieting) and sociocultural risk variables (e.g., differences in cultural proscriptions regarding the pursuit of thinness) in explaining the differential risk between men and women for BN (Striegel-Moore, Silberstein, \& Rodin, 1986). The role of gender in a risk model for BED has not yet been articulated clearly.

Gender differences in binge eating syndromes occur along several related dimensions. It is not clear whether the comparable rates of regular binge eating in women and men reflect a comparable risk for BED. A recent study found that men are less likely than women to report distress over binge eating (Lewinsohn et al., 2002), a symptom required for diagnosis of BED. This would suggest that even if men are as likely as women to engage in recurrent binge eating, men may be less likely to meet full-syndrome criteria for BED. Moreover, several studies have found markedly lower rates of extreme compensatory behaviors in men compared with women who binge eat (Hay, 1998; Lewinsohn et al., 2002; Woodside et al., 2001). When men develop disordered eating, they seem to be particularly likely to report recurrent binge eating.

Research has not examined comprehensively the clinical significance of recurrent binge eating, even in the absence of the requisite additional symptoms for a diagnosis of BED (or BN). One longitudinal study found that binge eating was associated with accelerated weight gain among women (Fairburn, Cooper, Doll, Norman, \& O'Connor, 2000). It is reasonable to assume that men who binge eat may also be at risk for weight gain. Although the health consequences of binge eating may be similar for men and women, men may be less likely to self-report their eating behavior in ways that would prompt its detection as symptomatic in survey studies.

\section{Ethnicity and BED}

The few studies that have examined ethnic group differences suggest that BED may be equally common among Black and White women, but these findings are based on selfreport data and need to be extended to other ethnic minority groups. Whether ethnic minority groups are more likely to develop BED than BN, as has been suggested by some experts (Smolak \& Striegel-Moore, 2001), still needs to be explored systematically. Results from the NEWHP showed that Black women are more likely than White women to report recurrent binge eating (Striegel-Moore et al., 2000), although it is unclear whether this finding would extend to rates of full-syndrome BED. Pike, Dohm, Striegel-Moore, Wilfley, and Fairburn (2001) evaluated the clinical presentation of BED in Black and White women. They found that Black women with BED had lower scores on the EDE Eating, Shape, Weight Concern, and Restraint scales than White women with BED. These ethnic differences in body image and dietary restraint are consistent with survey studies that have shown that Black women report less body image disturbance and are less likely to diet than White women (Smolak \& Striegel-Moore, 2001). More research is needed to determine whether these attitudinal and behavioral characteristics reflect ethnic differences in risk for eating disorders for which body image disturbance and extreme compensatory behaviors are defining features (Smolak \& Striegel-Moore, 2001; Striegel-Moore \& Smolak, 1996).

To date, the focus in epidemiologic studies has been solely on Blacks. Research is need to assess the prevalence of BED in additional groups. Hispanic women have comparable 
rates of binge eating to White women (Fitzgibbon et al., 1998) and similar high rates of obesity as Black women (Flegal, Caroll, Kuczmarski, \& Johnson, 1998), suggesting that BED may occur in this group with some frequency, yet the prevalence of BED in Hispanic individuals is not known. The scientific knowledge base about eating disorders in general and BED in particular among ethnic minority groups is so limited that any conclusions regarding ethnicity are premature.

\section{CONCLUSIONS}

Our review of prevalence studies illustrates the fact that epidemiologic research of BED has not progressed much. The studies reviewed offer at best a preliminary answer to the most basic question (how many people meet diagnostic criteria for BED?) and provide only a few clues regarding demographic indicators or other possible risk factors for BED. We caution, however, that the knowledge base from which these conclusions are reached is limited. For example, our review of the literature identified only one study, conducted in South Australia, that recruited a representative community sample of men and women and implemented a structured research interview for establishing the BED diagnosis (Hay, 1998). Reporting a point-prevalence rate of $1 \%$, this otherwise methodologically rigorous study unfortunately did not report whether BED was associated with gender. None of the studies measured the incidence (i.e., new cases) of BED. Studies are needed that examine the incidence and prevalence (including both current rates and lifetime rates) of $\mathrm{BED}$ in representative community samples and that employ interviewbased case finding methods.

Several tentative conclusions can be made on the basis of the existing research. The reports reviewed in the current study suggest that BED is more common than BN. In addition, the population of individuals with BED is more diverse than the population of individuals with BN in at least two ways. First, the gender difference in prevalence is less pronounced in BED than in BN. Second, relatively speaking, more minority women meet the criteria for BED than for BN. The significance of this demographic heterogeneity for an understanding of risk factors for BED has not yet been delineated conceptually or empirically. Future studies need to follow the path established by epidemiologic studies in $\mathrm{BN}$ and other mental disorders, including recruitment of representative samples of male and female populations that include ethnic minority groups. Diagnostic criteria need to be specified carefully and measured by research interview. Prospective risk factor studies that incorporate the role of gender and ethnicity are needed to improve our understanding of the etiology of BED.

\section{REFERENCES}

American Psychiatric Association. (1994). Diagnostic and statistical manual of mental disorders (4th ed.). Washington, DC: Author.

Beglin, S.J., \& Fairburn, C.G. (1992). What is meant by the term "binge"? American Journal of Psychiatry, 149, 123-124.

Bruce, B., \& Agras, W.S. (1992). Binge eating in females: A population-based investigation. International Journal of Eating Disorders, 12, 365-373.

Carter, W.P., Hudson, J.I., Lalonde, J.K., Pindyck, L., \& Pope, H.G. (2003). Pharmacologic treatments of binge eating disorder. International Journal of Eating Disorders, 34 (Suppl.), S74-S88.

de Zwaan, M. (2001). Binge eating disorder and obesity. International Journal of Obesity, 25 (Suppl. 1), S51-S55. 
Diez-Quevedo, C., Rangil, T., Sanchez-Planell, L., Kroenke, K., \& Spitzer R.L. (2001). Validation and utility of the patient health questionnaire in diagnosing mental disorders in 1003 general hospital Spanish inpatients. Psychosomatic Medicine, 63, 679-686.

Fairburn, C.G., \& Beglin, S.A. (1990). Studies of the epidemiology of bulimia nervosa. American Journal of Psychiatry, 147, 401-408.

Fairburn, C.G. \& Beglin, S.A. (1994). Assessment of eating disorder pathology: Interview or self-report questionnaire. International Journal of Eating Disorders, 16, 363-370.

Fairburn, C.G., \& Cooper, Z. (1993). The Eating Disorder Examination (12th ed.). In C.G. Fairburn \& G.T. Wilson (Eds.), Binge eating: Nature, assessment, and treatment (pp. 317-356). New York: Guilford Press.

Fairburn, C.G., Cooper, Z., Doll, H.A., Norman, P., \& O'Connor, M. (2000). The natural course of bulimia nervosa and binge eating disorder in young women. Archives of General Psychiatry, 57, 659-665.

Fairburn, C.G., Doll, H.A., Welch, S.L., Hay, P.J., Davies, B.A., \& O'Connor, M.E. (1998). Risk factors for binge eating disorder. Archives of General Psychiatry, 55, 425-432.

Fitzgibbon, M.L., Spring, B., Avellone, M.E., Blackman, L.R., Pingitore, R., \& Stolley, M.R. (1998). Correlates of binge eating in Hispanic, black, and white women. International Journal of Eating Disorders, 24, 43-52.

Flegal, K.M., Caroll, M.D., Kuczmarski, R.J., \& Johnson, C.L. (1998). Overweight and obesity in the United States: prevalence and trends, 1960-1994. International Journal of Obesity, 22, 39-47.

Garfinkel, P.E., Lin, E., Goering, P., Spegg, C., Goldbloom, D.S., Kennedy, S., Kaplan, A.S., \& Woodside, D.B. (1995). Bulimia nervosa in a Canadian community sample: Prevalence and comparison of subgroups. American Journal of Psychiatry, 152, 1052-1058.

Hay, P. (1998). The epidemiology of eating disorder behaviors: An Australian community based survey. International Journal of Eating Disorders, 23, 371-382.

Henderson, M., \& Freeman, C.P. (1987). A self-rating scale for bulimia. The 'BITE'. British Journal of Psychiatry, $150,18-24$.

Hoek, H.W. (2002). Distribution of eating disorders. In C.G. Fairburn \& K.D. Brownell (Eds.), Eating disorders and obesity (pp. 233-237). New York: Guilford Press.

Johnson, J.G., Spitzer, R.L., \& Williams, J.B. (1999). Health problems, impairment, and illnesses associated with bulimia nervosa and binge eating disorder among primary care and obstetric gynecology patients. Psychological Medicine, 31, 1455-1466.

Kendler, K.S., MacLean, C., Neale, M., Kessler, R., Heath, A., \& Eaves, L. (1991). The genetic epidemiology of bulimia nervosa. American Journal of Psychiatry, 148, 1627-1637.

Kessler, R.C., Zhao, S., Katz, S.J., Kouzis, A.C., Frank, R.G., Edlund, M., \& Leaf, P. (1999). Past-year use of outpatient services for psychiatric problems in the National Comorbidity Survey. American Journal of Psychiatry, 156, 115-123.

Kinzl, J.F., Traweger, C., Trefalt, E., Mangweth, B., \& Biebl, W. (1999a). Binge eating disorder in females: A population-based investigation. International Journal of Eating Disorders, 25, 287-292.

Kinzl, J.F., Traweger, C., Trefalt, E., Mangweth, B., \& Biebl, W. (1999b). Binge eating disorder in males: A population-based investigation. Eating and Weight Disorders, 4, 169-174.

Kraemer, H.C., Kazdin, A.E., Offord, D.R., Kessler, R.C., Jensen, P.S., \& Kupfer, D.J. (1997). Coming to terms with the terms of risk. Archives of General Psychiatry, 54, 337-343.

Lewinsohn, P.M., Hops, H., Roberts, R.E., Seeley, J.R., \& Andrews, J.A. (1993). Adolescent psychopathology: I. Prevalence and incidence of depression and other DSM-III-R disorders in high school students. Journal of Abnormal Psychology, 102, 133-144.

Lewinsohn, P.M., Seeley, J.R., Moerk, K.C., \& Striegel-Moore, R.H. (2002). Gender differences in eating disorder symptoms in young adults. International Journal of Eating Disorders, 32, 426-440.

Lewinsohn, P.M., Striegel-Moore, R.H., \& Seeley, J.P. (2000). The epidemiology and natural course of eating disorders in young women from adolescence to young adulthood. Journal of the American Academy of Child and Adolescent Psychiatry, 39, 1284-1292.

Linzer, M., Spitzer, R.L., Kroenke, K., Williams, J.B., Hahn, S., Brody, D., \& deGruy, F. (1996). Gender, quality of life, and mental disorders in primary care: Results from the PRIME-MD 1000 study. American Journal of Medicine, 101, 526-533.

Pike, K.M., Dohm, F.A., Striegel-Moore, R.H., Wilfley, D.D., \& Fairburn, C.G. (2001). A comparison of black and white women with binge eating disorder. American Journal of Psychiatry, 158, 1455-1460.

Smith, D.E., Marcus, M.D., Lewis, C.E., Fitzgibbon, M., \& Schreiner, P. (1998). Prevalence of binge eating disorder, obesity, and depression in a biracial cohort of young adults. Annals of Behavioral Medicine, 20, $227-232$.

Smolak, L., \& Striegel-Moore, R.H. (2001). Challenging the myth of the golden girl: Ethnicity and eating disorders. In R.H. Striegel-Moore \& L. Smolak (Eds.) Eating disorders: Innovative directions for research and practice (pp. 111-132). Washington, DC: American Psychological Association.

Spitzer, R.L., Devlin, M., Walsh, B.T., Hasin, D., Wing, R., Marcus, M., Stunkard, A., Wadden, T., Yanovski, S., Agras, S., Mitchell, J., \& Nonas, C. (1992). Binge eating disorder: A multisite field trail of the diagnostic criteria. International Journal of Eating Disorders, 11, 191-204.

Spitzer, R.L., Kroenke, K., \& Williams, J.B. (1999). Validation and utility of a self-report version of PRIME-MD. Journal of the American Medical Association, 282, 1737-1744.

Spitzer, R.L., Williams, J.B., Kroenke, K., Hornyak, R., \& McMurray, J. (2000). Validity and utility of the PRIMEMD Patient Health Questionnaire in assessment of 3000 obstetric-gynecologic patients: The PRIME-MD 
Patient Health Questionnaire Obstetrics-Gynecology Study. American Journal of Obstetrics and Gynecology, 183, 759-769.

Spitzer, R.L., Williams, J.B., Kroenke, K., Linzer, M., deGruy, F.V. 3rd, Hahn, S.R., Brody, D., \& Johnson, J.G. (1994). Utility of a new procedure for diagnosing mental disorders in primary care. The PRIME-MD 1000 study. Journal of the American Medical Association, 272, 1749-1756.

Spitzer, R.L., Yanovski, S., Wadden, T., Wing, R., Marcus, M.D., Stunkard, A., Devlin, M., Mitchell, J., Hasin, D., \& Horne, R.L. (1993). Binge eating disorder: Its further validation in a multi-site study. International Journal of Eating Disorders, 13, 137-154.

Striegel-Moore, R.H., \& Cachelin, F.M. (2001). Etiology of eating disorders in women. Journal of Counseling Psychology, 29, 635-661.

Striegel-Moore, R.H., Dohm, F.A., Pike, K.M., Wilfley, D.E., \& Fairburn, C.G. (2002). Abuse, bullying, and discrimination as risk factors for binge eating disorder. American Journal of Psychiatry, 159, $1902-1907$.

Striegel-Moore, R.H., Silberstein, L.R., \& Rodin, J. (1986). Toward an understanding of risk factors for bulimia. American Psychologist, 41, 246-263.

Striegel-Moore, R.H., \& Smolak, L. (1996). The role of race in the development of eating disorders. In L. Smolak, M. Levine, \& R.H. Striegel-Moore (Eds.), The developmental pychopathology of eating disorders: Implications for research, treatment, and prevention (pp. 259-284). Hillsdale, NJ: Erlbaum.

Striegel-Moore, R.H. \& Smolak, L. (2000). The influence of ethnicity on eating disorders in women. In R.M. Eisler \& M. Hersen (Eds.), Handbook of gender, culture, and health (pp. 227-254). Mahwah, NJ: Erlbaum.

Striegel-Moore, R.H., Wilfley, D.E., Pike, K.M., Dohm, F.A., \& Fairburn, C.G. (2000). Recurrent binge eating in black American women. Archives of Family Medicine, 9, 83-87.

Stunkard, A.J. (1959). Eating patters and obesity. Psychiatric Quarterly, 33, 284-295.

Thiels, C., \& Garthe, R. (2000). Praevalenz der Essstoerungen unter Studierenden [Prevalence of eating disorders in students]. Der Nervenarzt, 71, 552-558.

Walsh, B.T., \& Devlin, M.J. (1998). Eating disorders: Progress and problems. Science, 280, 1387-1390.

Wilfley, D.E., Wilson, G.T., \& Agras, W.S. (2003). The clinical significance of binge eating disorder. International Journal of Eating Disorders, 34 (Suppl.), S96-S106.

Williamson, D.A., Gleaves, D.H., \& Lawson, O.J. (1991). Biased perception of overeating in bulimia nervosa and compulsive binge eaters. Appetite, 13, 257-268.

Wonderlich, S.A., de Zwann, M., Mitchell, J.E., Peterson, C., \& Crow, S. (2003). Psychological and dietary treatments of binge eating disorder: Conceptual implications. International Journal of Eating Disorders, 34 (Suppl.), S58-S73.

Woodside, D.B., Garfinkel, P.E., Lin, E., Goering, P., \& Kaplan, A.S. (2001). Comparisons of men with full or partial eating disorders, men without eating disorders, and women with eating disorders in the community. American Journal of Psychiatry, 158, 570-574. 